Einführung zum Thema

Gastroenterologe 2021 · 16:65-67

https://doi.org/10.1007/s11377-021-00508-4

Angenommen: 12. Januar 2021

(c) Springer Medizin Verlag $\mathrm{GmbH}$, ein Teil von Springer Nature 2021

Die bedarfsgerechte Blutversorgung des Gastrointestinaltrakts und der Leber ist Grundvoraussetzung für die regelrechte Funktion dieser lebenswichtigen Organe. So banal diese Feststellung zur Bedeutung der arteriellen Blutzufuhr und zum venösen Abstrom für die abdominalen Organe ist, so schwierig ist es mitunter im klinischen Alltag, vaskuläre Erkrankungen in Gastrointestinaltrakt und Leber zu diagnostizieren, ätiologisch einzuordnen und dann adäquat zu behandeln. Anders als bei Durchblutungsstörungen des Herzens oder des zentralen Nervensystems können Symptome vaskulärer gastroenterologischer Erkrankungen sehr unspezifisch sein oder mitunter komplett fehlen. In diesen Fällen sind die aufmerksame Einordnung von auffälligen Laborbefunden, die zielgerichtete weitere Abklärung oder der differenzierte Einsatz bildgebender Verfahren entscheidend, um zur Diagnose zu gelangen. Mitunter sind „vaskuläre Erkrankungen des Gastrointestinaltrakts“ auch wichtige Hinweise auf eine andere zugrunde liegende (System-)Erkrankung; ein klassisches Beispiel hierfür wäre eine Pfortaderthrombose als Erstmanifestation einer myeloproliferativen Neoplasie.

Die therapeutischen Optionen vaskulärer gastroenterologischer Erkrankungen haben sich in den vergangenen Jahren deutlich erweitert. Dies betrifft zum einen neue medikamentöse Behandlungsverfahren wie z.B. Strategien zur Antikoagulation oder spezifische Innovationen wie Defibrotid beim sinusoidalen Obstruktionssyndrom. Zum anderen, vermutlich größeren Teil betrifft dies Weiter-

\author{
Frank Tacke' · Julia Mayerle ${ }^{2}$ \\ ${ }^{1}$ Medizinische Klinik m. S. Hepatologie und Gastroenterologie, Campus Virchow-Klinikum (CVK) und \\ Campus Charité Mitte (CCM), Charité Universitätsmedizin Berlin, Berlin, Deutschland \\ ${ }^{2}$ Klinik und Poliklinik für Innere Medizin II, Campus Innenstadt/Großhadern, LMU Klinikum, München, \\ Deutschland
}

\title{
Vaskuläre Erkrankungen in Gastrointestinaltrakt und Leber
}

entwicklungen in interventionell-radiologischen und chirurgischen Verfahren. Umgekehrt bedeutet dies, dass vaskuläre Erkrankungen in Gastrointestinaltrakt und in der Leber grundsätzlich interdisziplinär diskutiert und behandelt werden sollten. Mit der vorliegenden Ausgabe von Der Gastroenterologe möchten wir anhand aktueller Übersichtsartikel zu wichtigen Krankheitsbildern den derzeitigen Stand der Diagnostik und Therapie darstellen und konnten hierfür renommierte Expertinnen und Experten aus führenden Zentren gewinnen.

Durchblutungsstörungen mit Minderperfusion in den mesenterialen Versorgungsgebieten manifestieren sich klassischerweise als ischämische Enterokolitis. Lukas Neuhaus und Helga Török zeigen die vielfältigen klinischen Manifestationen und die zugrunde liegenden Mechanismen auf. Grundsätzlich sind nichtokklusive von okklusiven Gefäßveränderungen $\mathrm{zu}$ unterscheiden, was entscheidende Konsequenzen für die Prognose und das passgenaue Therapieverfahren hat. Okklusive mesenteriale Ischämien, besonders bei gangränösem Verlauf, müssen sofort als Notfälle erkannt und unmittelbar einer Therapie, in der Regel chirurgisch oder endovaskulär-interventionell, zugeführt werden. Die nichtokklusive ischämische Kolitis hat dagegen einen gutartigeren Verlauf, sodass konservative Maßnahmen oftmals ausreichen.

Die Pfortaderthrombose tritt nicht nur bei Leberzirrhose oder Leberzellkarzinom auf, sondern kann auch Erstmanifestation einer Thrombophilie oder myeloproliferativen Erkrankung sein.
Christian Steib und Kollegen stellen den aktuellen diagnostischen Algorithmus bei Verdacht auf Pfortaderthrombose dar. In diesem Artikel wird deutlich, dass das therapeutische Management immer interdisziplinär festgelegt werden sollte. Neben Behandlung der Grundkrankheit und Antikoagulation bieten Chirurgie und interventionelle Radiologie neue Optionen für eine Thrombektomie, Rekanalisation oder Shuntverfahren.

Gefürchtete Folge einer Pfortaderthrombose ist die portale Hypertension mit allen Komplikationen (wie z.B. Ösophagusvarizenblutung), die aus der Behandlung von Patienten mit Leberzirrhose gut bekannt sind. Eine besondere differenzialdiagnostische Herausforderung ist jedoch, wenn eine portale Hypertension ohne gleichzeitige bestehende Leberzirrhose auftritt. Mit dem „bunten" Krankheitsbild der nichtzirrhotischen portalen Hypertension (NCPH) beschäftigt sich der Artikel von Alexander Queck und Jonel Trebicka. Bei der $\mathrm{NCPH}$ ist die Ursachenidentifikation mitunter schwierig, aber wichtige Differenzialdiagnosen, wie Schistosomiasis, Budd-Chiari-Syndrom, Medikamententoxizität (z.B. Azathioprineinnahme), hämatologische Erkrankungen, Amyloidose oder Sarkoidose, müssen sorgfältig abgeklärt werden. In der Therapie spielen die Behandlung der Grunderkrankung und die Prophylaxe von Komplikationen der NCPH die entscheidende Rolle für die langfristige Prognose der Patienten.

Einen Sonderfall von erheblicher, lebensbedrohlicher Relevanz stellt das sinusoidale Obstruktionssyndrom (SOS), auch als „veno-occlusive disease“ (VOD) 
bezeichnet, dar. Dieses Syndrom beschreibt eine seltene, vor allem nach Stammzelltransplantation auftretende, potenziell lebensbedrohliche Zirkulationsstörung der hepatischen Sinusoide. Marten Schulz und Alexander Wree zeigen in ihrem Übersichtsartikel auf, dass die Diagnose des SOS neben klinischen Kriterien durch nichtinvasive Bildgebungstechniken entscheidend unterstützt werden kann. Da die Therapieoptionen bei SOS mit zumeist schwerer hämatologischer Grunderkrankung limitiert sind (für schwere Verläufe steht Defibrotid zur Verfügung), haben die Prophylaxe und die Früherkennung einen besonderen Stellenwert.

Vaskuläre Erkrankungen mögen uns Gastroenterologen im Alltag selten erscheinen, in ihrer Summe und in ihrer Vielfalt sind sie aber wichtige (und nicht so seltene!) Differenzialdiagnosen beiunspezifischen Symptomen (Bauchschmerzen, Durchfälle, Aszites) oder bei Laborauffälligkeiten (Leberwerterhöhung, Laktatämie). Für die Diagnostik der einzelnen Krankheitsbilder existieren aktuelle Algorithmen, die optimale Therapie sollte interdisziplinär festgelegt werden. Hierbei muss immer berücksichtigt werden, ob die vaskuläre Erkrankung allein auf den Gastrointestinaltrakt bzw. die Leber beschränkt ist oder ob sie vielmehr eine Systemerkrankung widerspiegelt.

\section{Korrespondenzadresse}

Prof. Dr. Frank Tacke
Medizinische Klinik m.
S. Hepatologie und
Gastroenterologie, Campus
Virchow-Klinikum (CVK) und
Campus Charité Mitte (CCM),
Charité Universitätsmedizin
Berlin
Augustenburger Platz 1,
13353 Berlin, Deutschland
frank.tacke@charite.de

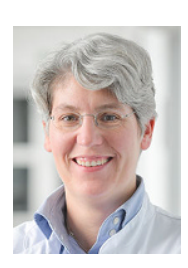

Prof. Dr. Julia Mayerle Klinik und Poliklinik für Innere Medizin II, Campus Innenstadt/Großhadern, LMU Klinikum

Marchioninistraße 15, 81337 München, Deutschland julia.mayerle@med.unimuenchen.de
Interessenkonflikt. F. Tacke und J. Mayerle geben an, dass kein Interessenkonflikt besteht.
Machen Sie sich fit mit dem „Facharzt-Training Innere Medizin“!

Bereiten Sie sich auf die Facharztprüfung vor oder möchten Sie Ihr fachspezisches Wissen mit typischen Fallbeispielen aus der Inneren Medizin auffrischen? Dann sind die Sonderhefte von Der Internist "FacharztTraining Innere Medizin" genau das Richtige für Sie.

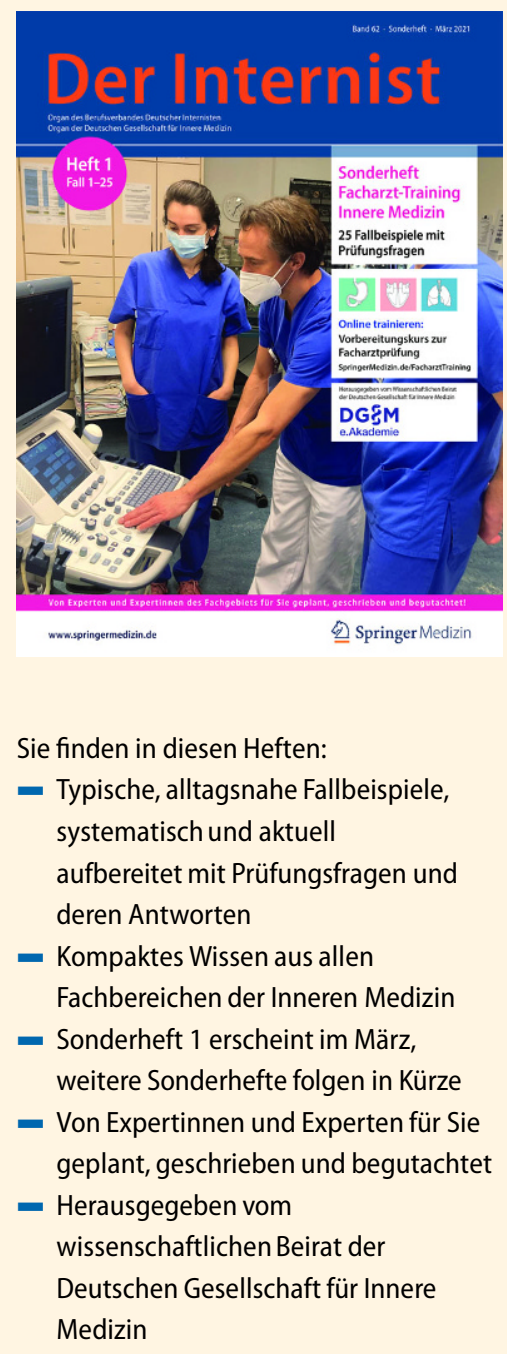

Bestellen Sie „Facharzt-Training Innere Medizin" Sonderheft 1 zum Preis von 44 Euro unter Angabe des Aktionscodes C0019640 bei Marie-Luise.Witschel@springer.com 
Hier steht eine Anzeige.

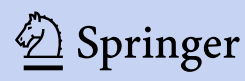

